\title{
Experimental BSM prospects at the HL-LHC
}

\section{Kerstin Hoepfner ${ }^{1}$ on behalf of the CMS and ATLAS collaborations}

RWTH Aachen, III. Phys. Inst. A

Otto-Blumenthal-Str., Aachen, Germany

E-mail: hoepfnerephysik.rwth-aachen.de

This paper presents examples from the wealth of physics studies to determine the achievable sensitivity at the high-luminosity LHC. Beyond-the-standard-model (BSM) searches concern new heavy bosons, supersymmetric models, exotic signatures with displaced leptons and many more.

Sixth Annual Conference on Large Hadron Collider Physics (LHCP2018)

4-9 June 2018

Bologna, Italy

\footnotetext{
${ }^{1}$ Speaker
} 


\section{What means high luminosity?}

This paper presents physics studies for the future operation of the LHC collider with highluminosity (HL-LHC), also called LHC Phase-2. High luminosity means about five times higher instantaneous luminosity, with $40 \mathrm{MHz}$ operation (one BX every $25 \mathrm{~ns}$ ) at $\sqrt{s}_{s}=14 \mathrm{TeV}$. This yields challenging conditions for the CMS detector: up to 200 overlaying (pileup) events, high rates and high radiation levels, especially in the forward regions. The total accumulated luminosity is anticipated to reach $3000 / \mathrm{fb}$ of data, corresponding to $10 \mathrm{x}$ the data expected at the end of Phase-1. This environment offers great physics opportunities: e.g. discover new particles (e.g. dark matter, heavy resonances, the ,unexpected"), access weak couplings and rare processes. If discovered, particle properties (mass, couplings, spin, etc.) will be studied.

\section{Searches for new particles}

Several benchmark analyses searching for new particles will continue at the HL-LHC. One prominent example is the search for narrow dijet resonances which could originate from excited quarks $\left(\mathrm{q}^{*}\right)$, heavy bosons or quantum black holes [1]. The so-called bump-hunter algorithm - scanning for a bump in the steeply falling $\mathrm{M}_{\mathrm{jj}}$ mass spectrum is a model-independent technique applied in several analyses. With the 3000/fb of the HL operation, exclusion limits of $8 \mathrm{TeV}$ for $\mathrm{q}^{*}$ and $10 \mathrm{TeV}$ for quantum black holes can be reached.

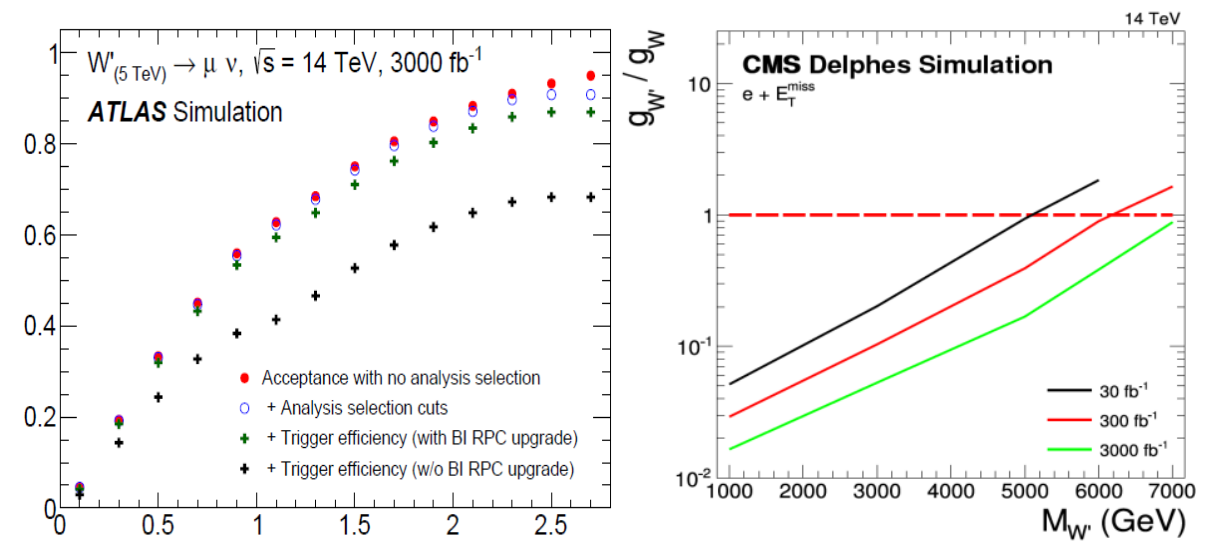

Figure 1 Searches for heavy resonances, such as $W^{\prime}$, are important benchmark channels to evaluate the detector performance as well as physics sensitivity. Left: Increase in trigger efficiency for the process $W^{\prime} \rightarrow \mu v$ for the ATLAS Phase-2 upgrade with additional RPC detectors. Right: Weak W'couplings $\left(\mathrm{g}_{\mathrm{W}} / \mathrm{g}_{\mathrm{W}}\right)$ can be studied with high luminosity.

New heavy bosons (neutral as $\mathrm{Z}^{\prime}$, charged as $\mathrm{W}^{\prime}$ ) are predicted in many standard model (SM) extensions and important benchmarks for the ATLAS and CMS experiments. Not only in terms in physics sensitivity but also as a reference for detector performance for high momentum particles. Fig.1-left shows the cumulative acceptance for the process $\mathrm{W}^{\prime} \rightarrow \mu \nu$ as a function of the pseudorapidity $(\eta)$ for the upgraded detector [2]. With the installation of additional RPC detectors in Phase-2, the trigger efficiency will increase from $70 \%$ to $90 \%$. 
With high luminosity processes with small cross sections and weak couplings can be studied, as depicted in Fig.1-right for the process $\mathrm{W}^{\prime} \rightarrow \mathrm{ev}$. The coupling strength for a $\mathrm{W}^{\prime}$ boson is expressed as a ratio $\mathrm{g}_{\mathrm{W}} / \mathrm{g}_{\mathrm{W}}$ with respect to the $\mathrm{SM} \mathrm{W}$ boson. [3]

\section{Where is SUSY?}

The present LHC has excluded large parts of the natural SUSY parameter space; limits for strong SUSY production are above $1 \mathrm{TeV}$ and top and bottom squarks are already highly constrained. There are still many opportunities in the electroweak sector though which may be the dominating sector if squarks and gluinos are heavy. In most SUSY breaking scenarios EWK-inos are expected to be of the order $100 \mathrm{GeV}$ in mass based on naturalness. Given that EWK-inos are produced via EWK production the cross sections are small and HL-LHC has a large potential to increase sensitivity.

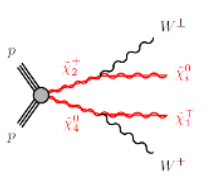

A brand-new CMS study for EWK-inos exploits the striking signature of same-sign leptons. It searches for mass degenerate $\tilde{X}_{2}^{ \pm} \tilde{X}_{4}^{0}$ which can have a large cross section to same-sign WW and Higgsinos which are taken to be the lightest SUSY particles. Since the mass difference is small, the $\mathrm{W}$ bosons are soft yielding very low $\mathrm{p}_{\mathrm{T}}$ leptons. This constitutes a challenge to the trigger and their detection. In a binned $\mathrm{m}_{\mathrm{T}}$ spectrum as shown with seven mass bins in Fig.2-left, a potential signal could be extracted. If nothing is found, EWK-ino masses up to $900 \mathrm{GeV}$ can be excluded with 3000/fb (see Fig.2-right) [4].
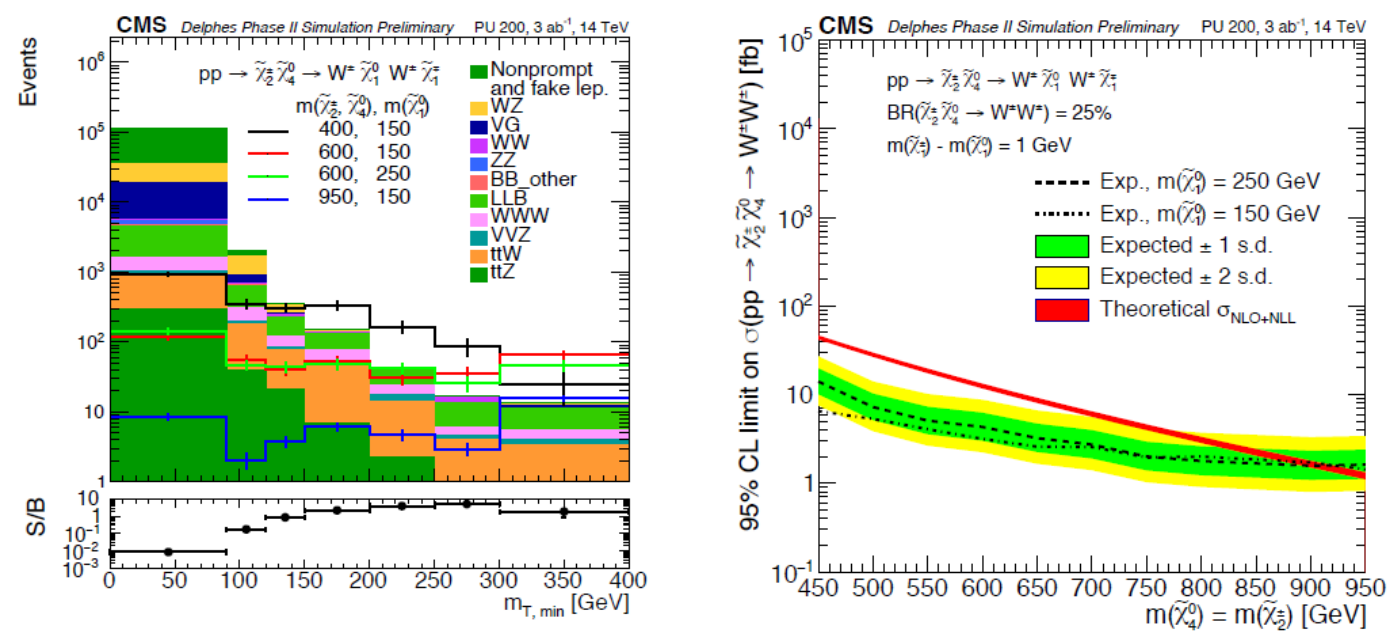

Figure 2 Search for EWK-inos exploiting the striking signature of same-sign leptons. Left: The discriminating variable $m_{T, m i n}$ reconstructed from the leptons and missing transverse energy. The seven bins would allow an extraction of the signal from the background. Right: Exclusion limit in case of no obervation of a signal.<smiles>CC1(C)CCC2([P+](C)(C)C)CC12C</smiles>

In other models, direct production of stau pairs can become the dominant EWK production mechanism. This ATLAS [5] study assumes a $100 \%$ BR to tau (using the dominating hadronic decay mode) and neutralino (yielding large MET). The signal region is defined at large transverse masses (see Fig.3-left). The resulting sensitivity after full event selection is displayed in Fig.3-right for both, the five 
sigma discovery sensitivity and the $95 \%$ exclusion. Studies with $20 \%, 30 \%$ and $50 \%$ systematics on the backgrounds are done, showing no significant impact on sensitivity.
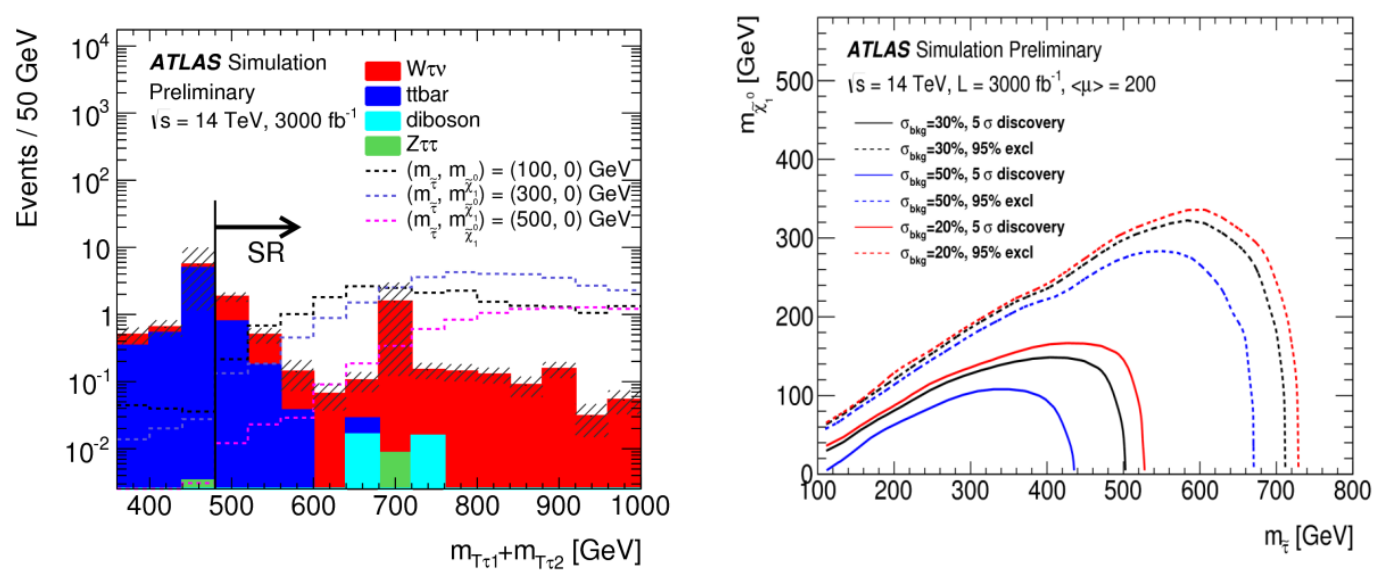

Figure 3 Direct production of stau pairs, based on the selection of two opposite-sign taus, loose jet and Zveto and MET>280 GeV. Left: The signal region (SR)defined in $m_{T}(\tau 1)+m_{T}(\tau 2)$ Right: Discovery and exclusion reach for different assumptions on the background precision.

\section{Exotic signatures}

Since it is not known how new physics will look like, it is essential to be sensitive to all possible signatures. New physics could manifest itself with a non-standard signature, requiring dedicated trigger and reconstruction algorithms and specific detector features. One example are slow moving particles. One way to detect such particle is their anomalous $\mathrm{dE} / \mathrm{dx}$ [3] provided they are triggered.
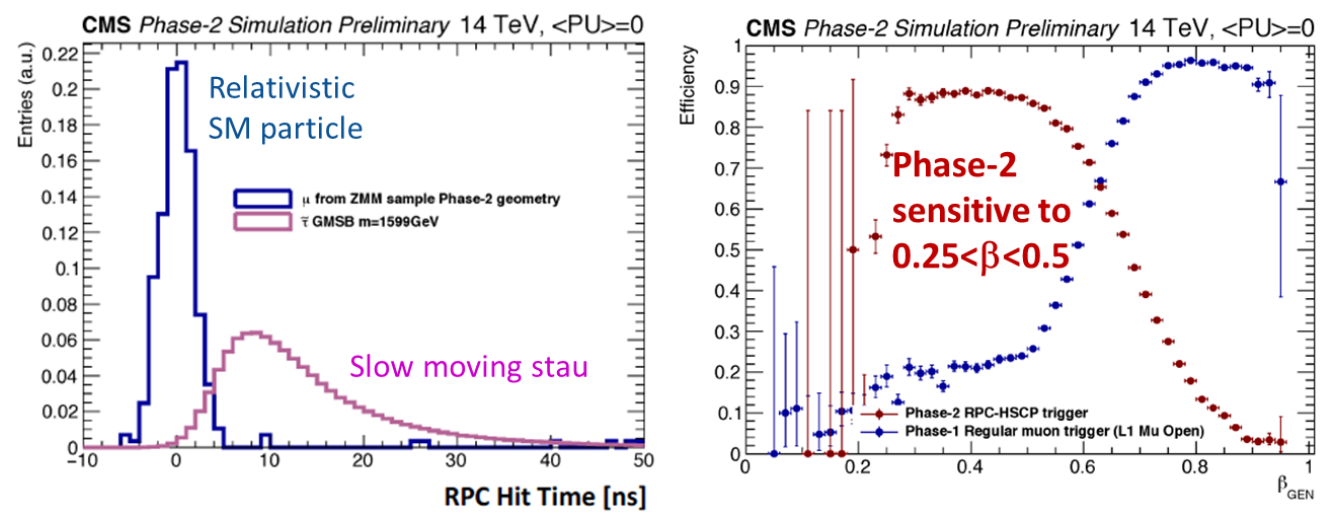

Figure 4 Triggering muons from the decay of slow moving particles, e.g. from a SUSY tau (stau). Left:Muon hits from such decays will appear later than hits from relativistic SM particles. Right:Phase-2 sensitivity for slow-moving particles (represented by low $\beta$ ) in comparison to Phase-1.

Muons from the decay of slow moving particles can already be identified at the trigger level based on the difference in arrival time with respect to relativistic SM particles. The muons from such decays are delayed w.r.t. $\mathrm{BX}=0$ and will spill over in neighbouring BXs. Fig.4-left illustrates such a time difference as seen by CMS RPC chambers instrumenting the barrel and 
the forward muon system. The anticipated ns time resolution of the upgraded muon trigger allows to detect slowly moving charged particles by their hits. This will significantly extend the trigger capability to very slow particles with beta $<0.5$ (red) compared to present conditions, as displayed in Fig.4-right [6].

In GMSB SUSY models, smuons can be the co-NLSP (= next to lightest LSP) and almost degenerate in mass. They decay to a SM muon and a gravitino (= causing MET) with lifetimes which can be very long such that the displacement is of the order (meter) and trigger and reconstruction has to be performed by the muon system in stand-alone, without the tracker. This study [6] evaluated the sensitivity of a dedicated displaced reconstruction scenario in terms of smuon mass (Fig.5-left) and decay length (Fig.5-right).
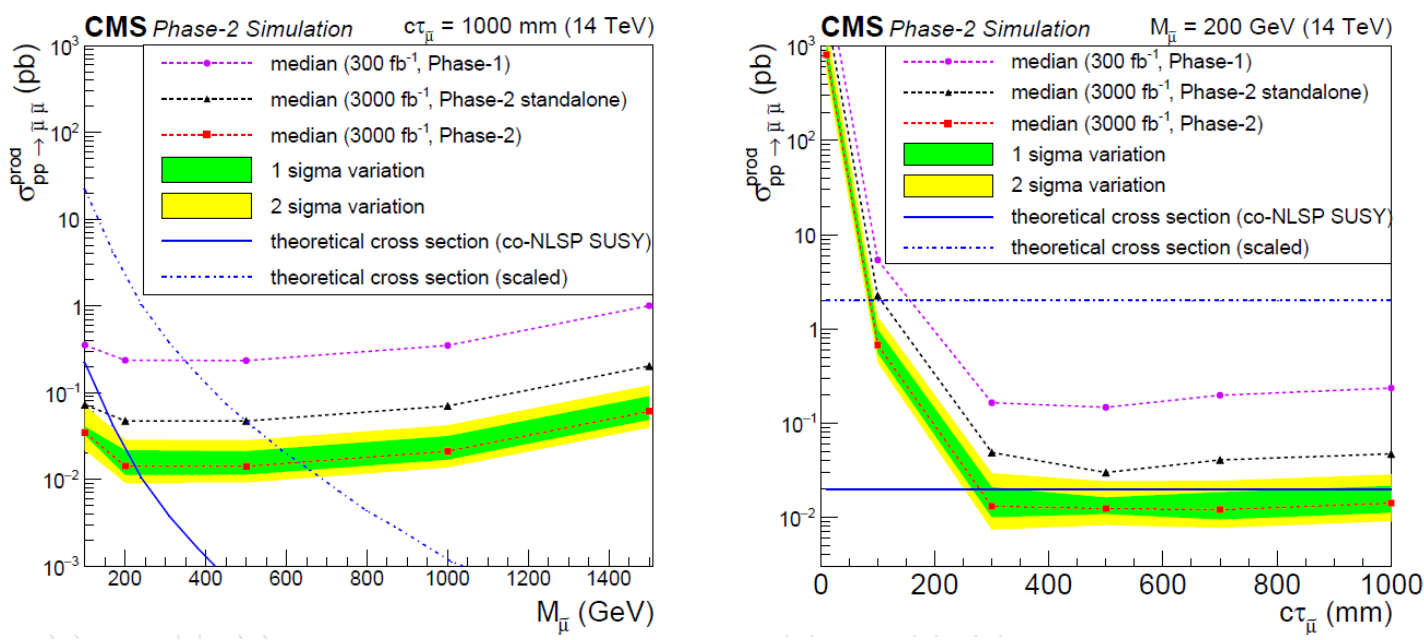

Figure 5 Sensitivity for displaced muons in terms of smuon mass (left) in a GMSB SUSY model where the smuon in the co-NLSP. On the right, the sensitivity of the production cross section as a function of c $\tau$ representing the decay length.

\section{Searches related to observed flavour anomalies}

Possible explanations for the observed flavour anomalies include the prediction of $\mathrm{Z}^{\prime}$-like mediators or leptoquark (LQ)-like mediators. TeV-scale masses and the 3rd generation are favoured. Complementary searches for heavy bosons with special emphasis on final states containing top and/or bottom quarks are interesting in this context. Fig.6 shows extrapolations of existing run-2 analyses to HL statistics considering scenarios of systematic uncertainties as shown in the figure legend.

The decay $\mathrm{W}^{\prime} \rightarrow \mathrm{tb} \rightarrow \mathrm{e} / \mu+\mathrm{b}$-jets [7] allows, for example, to probe scenarios such as $\mathrm{m}\left(v_{\mathrm{R}}\right)>$ $\mathrm{m}\left(\mathrm{W}^{\prime}{ }_{\mathrm{R}}\right)$, which cannot be studied with leptonic $\mathrm{W}^{\prime}$ searches. The knowledge of systematic uncertainties impacts the physics reach, as shown in Fig.6-left. One expects improvements on the theoretical knowledge of higher order corrections, while detector understanding and datadriven methods will profit from larger statistics.

In Fig.6-right, the projected exclusion limit at HL-LHC for the channel $\mathrm{Z}^{\prime} \rightarrow \mathrm{tt} \rightarrow \mathrm{WbWb} \rightarrow$ lvbqqb is shown [8]. For this analysis the selection steps are derived from run-2, although simplified. The discriminating variable is the mass of the ttbar system. Two categories are included: resolved and boosted, depending on the $\mathrm{Z}^{\prime}$ mass. 

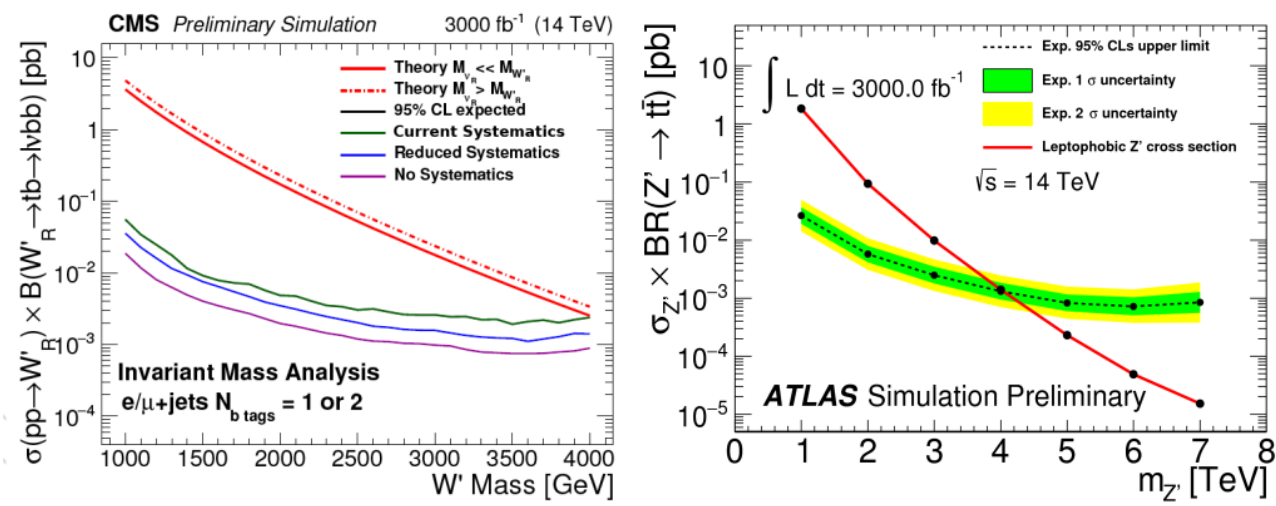

Figure 6 Projected exclusion limits from run-2 analyses with different systematics scenarios. Left: $W^{\prime} \rightarrow t b \rightarrow e / m u+b$-jets. Right: Exclusion limit for $Z^{\prime} \rightarrow t t \rightarrow W b W b \rightarrow l v b q q b$ for $3000 / f b$.

\section{Summary}

We have shown selected examples of the rich BSM potential at the HL-LHC. Many more studies are ongoing, from simple projections of a run-2 result up to a number of full analyses. Besides a variety of benchmark analyses, new models, lower cross sections and weaker couplings are studied. The expected high luminosity will provide a rich harvest and hopefully allow another discovery.

\section{References:}

[1] ATL-PHYS-PUB-2015-004: Dijet resonance searches with the ATLAS detector at 14 TeV LHC

[2] CERN-LHCC-2017-017 ATLAS Muon upgrade TDR

[3] CMS-PAS-EXO-14-007: Enhanced scope of a Phase 2 CMS detector for the study of exotic signatures at the HL-LHC

[4] CMS-TDR-17-007 CMS Forward calorimeter TDR

[5] ATL-PHYS-PUB-2016-021 Direct production of stau pairs

[6] CMS-TDR-17-003 CMS Muon upgrade TDR

[7] CMS-PAS-FTR-16-005: Estimated Sensitivity for New Particle Searches at the HL-LHC

[8] ATL-PHYS-PUB-2017-002: Study on the prospects of a $t t^{-}$resonance search in events with one lepton at a High Luminosity LHC

[9] CMS-TDR-17-006 CMS ECAL barrel TDR 